\title{
Extraction of high-intensity ion beams from a laser plasma by a pulsed spherical diode
}

\author{
Yoshiyuki Oguri, ${ }^{*}$ Ken-ichi Kashiwagi, ${ }^{\dagger}$ Jun-ichi Kaneko, Jun Hasegawa, Masahiro Yoshida, ${ }^{\ddagger}$ and Masao Ogawa \\ Research Laboratory for Nuclear Reactors, Tokyo Institute of Technology, O-okayama 2-12-1-N1-14, \\ Meguro-ku, 152-8550 Tokyo, Japan
}

(Received 2 March 2005; published 24 June 2005)

\begin{abstract}
High-current $\mathrm{Cu}^{+}$ion beams were extracted from a laser-produced plasma using a pulsed high-voltage multiaperture diode driven by an induction cavity. The amplitude and the duration of the extraction voltage were $130 \mathrm{kV}$ and $450 \mathrm{~ns}$, respectively. During the extraction, explosive beam divergence due to the strong space-charge force was suppressed by the focusing action of the gap between concentric hemispheres. Modulation of the extracted beam flux due to the plasma prefill in the gap has been eliminated by using a biased control grid put on the anode holes. By means of this extraction scheme we obtained a rectangular beam pulse with a rise time as short as $\approx 100 \mathrm{~ns}$. The beam current behind the cathode was limited to $\approx 0.1 \mathrm{~A}$, owing to space-charge effects, as well as to poor geometrical transmission through the cathode sphere. From the measurement of the extracted beam current density distribution along the beam axis and the beam profile measurement, we found a beam waist slightly downstream of the spherical center of the diode structure. The measured beam behavior was consistent with numerical results obtained via a 3D particle code. No serious degradation of the beam emittance was observed for the grid-controlled extraction scheme.
\end{abstract}

DOI: $10.1103 /$ PhysRevSTAB.8.060401

PACS numbers: 52.59. $-\mathrm{f}, 41.85 .-\mathrm{p}, 52.58 . \mathrm{Hm}$

\section{INTRODUCTION}

In scenarios of heavy-ion inertial-confinement fusion (HIF) based on induction linacs, ion sources for beam drivers are required to supply $10^{13}-10^{14}$ ions during $\approx$ $20 \mu$ s to the accelerator system $[1,2]$. The corresponding beam current is $0.1-1$ particle amperes. Very heavy ions $(A>200)$ with low charge states $(1-3+)$ are preferable for reduction of space-charge effects. The normalized emittance should be as small as $\approx 1 \pi \mathrm{mm}$ mrad. Such high-current beams are available only by pulsed operations. Moreover, rectangular beam pulses with a fast rise time $(<100 \mathrm{~ns})$ are needed for stable beam optics under strong space-charge force, and for the efficient use of "volt $\times$ second" of the given induction cores [3]. Beams with a rectangular waveform can be extracted from a pulsed plasma by a pulsed extraction voltage which has a shorter duration than that of the plasma blow. In this case, higher extraction voltages are advantageous in obtaining a higher beam current. However, since the gap is filled with the plasma before the extraction voltage is applied, the ions are extracted from an ion-emitting surface which is moving in the gap toward the anode. Hence the beam optics and the beam current waveform are expected to be unstable in such a "plasma prefill" mode [4-6]. In order to eliminate such a

\footnotetext{
*Electronic address: yoguri@nr.titech.ac.jp

${ }^{\dagger}$ Present address: Ninja Systems, Inc., Toyotama-kami 2-25-9, Nerima-ku, 176-0011 Tokyo, Japan.

${ }^{\ddagger}$ Present address: High Energy System Development, Energy System Division, Ishikawajima-Harima Heavy Industries Co., Ltd., Shin-nakahama-cho 1, Isogo-ku, 235-8501 Yokohama, Japan.
}

beam-extraction mode, one can apply a negative bias voltage on a fine metallic mesh attached to the anode $[7,8]$.

To obtain a well-focused beam, the ion-emitting surface of the plasma should have a slightly concave shape in the extraction hole. If the plasma flux is too high, however, the plasma inflates into the gap, making the plasma surface a convex shape. Moreover, due to the bulge of the plasma, the effective gap length can be reduced, leading to serious mismatching in the beam-extraction optics. Owing to the space-charge force, a high-current beam can rapidly diverge as soon as it is extracted from the source plasma. This effect leads to a large beam loss and mismatching to the next accelerator section, which suggests the beam extraction should be accompanied by a focusing effect, as in the case of extraction from a plasma meniscus. However, formation of a stable meniscus is difficult, especially for pulsed plasmas. A multiaperture anode with a concave shape can also form a similar electric field distribution in the gap, which is suitable for beam focusing. If the bulge of the source plasma is still large, the shape of the ion-emitting surface in each hole must be controlled again by a mesh grid attached to each hole.

Laser-produced plasmas are possible sources of pulsed, high-intensity ion beams. Ion sources using such a laser plasma (laser ion source) have been studied thus far by many scientists in order to produce ions with very high charge states $>+10$ [9-11]. However, by adjusting the laser flux, not only highly charged ions but also ions with low charge states are made available [12,13]. In this paper we present experimental results on the extraction of high current, singly charged ions from a laser-produced plasma by a pulsed high voltage over $100 \mathrm{kV}$. We investigate the effect of the negative bias voltage applied on the anode 
grid. Beam focusing by a spherical multiaperture diode structure is demonstrated. The experimental results of the focusing effect under the strong space-charge force are numerically analyzed by a 3D particle code.

\section{EXPERIMENTAL METHOD}

The experimental setup is illustrated in Fig. 1. The source plasma was produced by irradiating a copper block with a Nd:YAG laser. To increase the fraction of singly charged ions of $\mathrm{Cu}^{+}$, we used the second harmonic $(\lambda=$ $532 \mathrm{~nm}$ ) of this laser [12]. The duration and the maximum energy of the laser were $6 \mathrm{~ns}$ and $50 \mathrm{~mJ}$, respectively. The laser was focused onto the target by a plano-convex lens with a focal length of $150 \mathrm{~mm}$. The incident angle to the target surface was $45^{\circ}$. The focal spot size on the $\mathrm{Cu}$ target was $1.5 \mathrm{~mm}$ (horizontal) $\times 1 \mathrm{~mm}$ (vertical). The corresponding flux was $\approx 10^{8}-10^{9} \mathrm{~W} / \mathrm{cm}^{2}$. To increase the duration of the plasma blow and to reduce the ion flux at the extraction surface, the plasma was expanded in a cylindrical chamber having a length of $100 \mathrm{~mm}$. After passing through this expansion chamber, the plasma reaches the anode. The plasma ion flux at the anode position was measured by a biased Faraday cup with a $\phi 3-\mathrm{mm}$ aperture. The diode structure was detached during this measurement. Under the condition above, the charge state of copper ions at the anode position was mostly 1+ [6].

To focus the beam under the strong space-charge force during extraction, we applied a diode structure with a spherical configuration, which consists of a pair of stainless-steel hemispherical shells. Figure 2 shows the electrodes of the diode. The radii of the anode and the cathode shell were 50 and $25 \mathrm{~mm}$, respectively. On each shell, 19 beam holes were drilled in a regular hexagonal pattern. The holes on the anode are $8 \mathrm{~mm}$ in diameter with a $10 \mathrm{~mm}$ pitch. The effective area of the ion-emitting surface $S_{\text {eff }}$ was $20 \mathrm{~cm}^{2}$. This area was evaluated by calculating a hexagonal area which covers all 19 holes on the



FIG. 1. Setup for the beam extraction and focusing experiment.
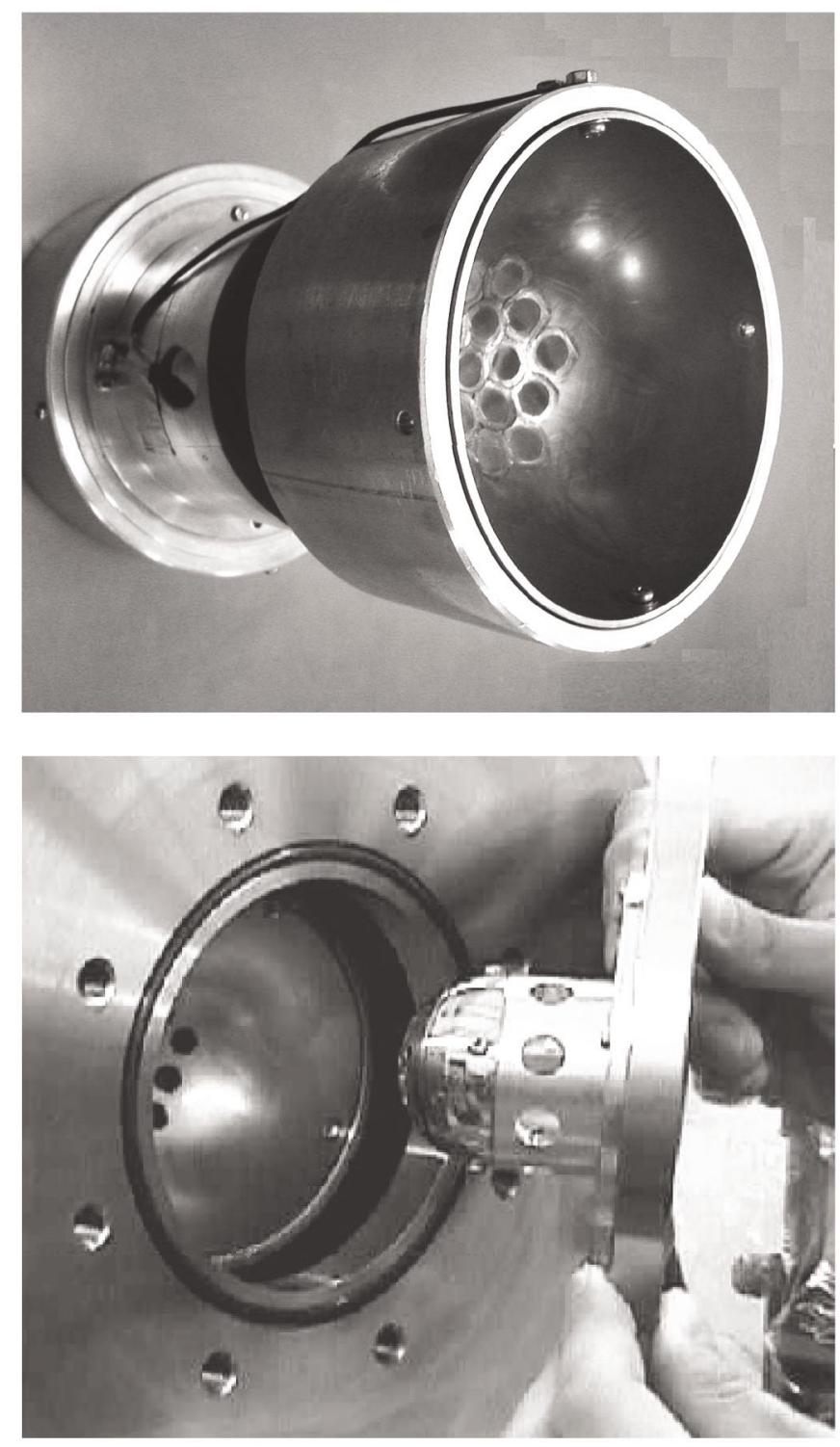

FIG. 2. Hemispherical anode with mesh grid (upper) and the cathode during assembly of the diode (lower).

anode sphere. For the cathode, the hole diameter and the pitch are 4 and $5 \mathrm{~mm}$, respectively.

In order to control the plasma flow into the gap, the anode was negatively biased with respect to the $\mathrm{Cu}$ target and the plasma expansion chamber. Bias voltages up to $-90 \mathrm{~V}$ were supplied by a dry battery. Diffusion of the plasma through the anode holes was eliminated by a metallic mesh put over each anode hole. We used stainlesssteel woven wire meshes with a square configuration (250 mesh/inch, wire diameter $=35 \mu \mathrm{m})$. No mesh grid was attached on the cathode. The total ion current into the gap was measured by a biased planar $(\phi 50 \mathrm{~mm})$ charge collector inserted between the anode and the cathode. The averaged ion current density $J_{\mathrm{av}}$ behind the anode is defined by the measured total ion current divided by $S_{\text {eff }}$.

The extraction voltage was applied to the anode by an induction accelerator module $[6,12,14]$, where a pulsed 
current around a magnetic core induced the high voltage in the gap. The driving current was delivered by a pulse forming line. The amplitude and duration of the induction voltage were $130 \mathrm{kV}$ and $450 \mathrm{~ns}$, respectively. The dry battery to supply the anode bias voltage was accommodated in the high-voltage terminal of the induction cavity.

Behind the cathode, waveforms of the extracted beam current density were measured with a biased, axially movable $50-\Omega$ Faraday cup with a $\phi 2-\mathrm{mm}$ aperture. Timeaveraged transverse beam profiles were measured using burn paper (Kodak Linagraph direct print) at different longitudinal positions. Beam emittance was measured by a pepper-pot method [14] also using this thermal paper.

\section{NUMERICAL METHOD}

Since the behavior of high-current beams from the hexagonal multiaperture structure is far from axial symmetry, one needs a full 3D numerical calculation for detailed beam dynamics analysis. In order to explain the experimental results of the beam extraction and focusing effects, behavior of the high-current ion beams during the acceleration and transport were analyzed by using a 3D particle code. In this computer code, the equation of motion for each macroparticle was integrated by means of a leap-frog method.

For the gap between the anode and the grounded cathode, we used a simple electrostatic potential between the concentric spheres given by

$$
U(r)=V\left(\frac{R_{\mathrm{a}}}{R_{\mathrm{a}}-R_{\mathrm{c}}}\right)\left(1-\frac{R_{\mathrm{c}}}{r}\right), \quad\left(R_{\mathrm{c}}<r<R_{\mathrm{a}}\right),
$$

where $V$ is the gap voltage and $r$ is the radial coordinate in the spherical geometry. The anode and cathode radius are denoted by $R_{\mathrm{a}}$ and $R_{\mathrm{c}}$, respectively.

The space-charge force was calculated directly by summing up the electrostatic forces from all other macroparticles. We used 6000 macroparticles for this simulation. Image-charge force was not taken into account. These macroparticles were homogeneously injected into the gap through 19 anode holes during the pulse duration of $450 \mathrm{~ns}$. Drift velocity of the plasma ( $\approx 130 \mathrm{eV}$ for $\mathrm{Cu}^{+}$ions) in the $z$ direction (beam axis) was taken into account in the initial condition of the calculation. The lateral ( $x$ and $y$ direction) velocity due to the thermal motion of ions was neglected because it was much smaller than the drift energy. That is, the initial emittance of each beamlet is zero in this calculation. The deflection effect due to acceleration by the negatively biased mesh grid was included in the beam-optics calculation by using Snell's law. Beam loss due to the collision with the cathode sphere was also included in the calculation, taking the hole size and positions of the cathode into account.

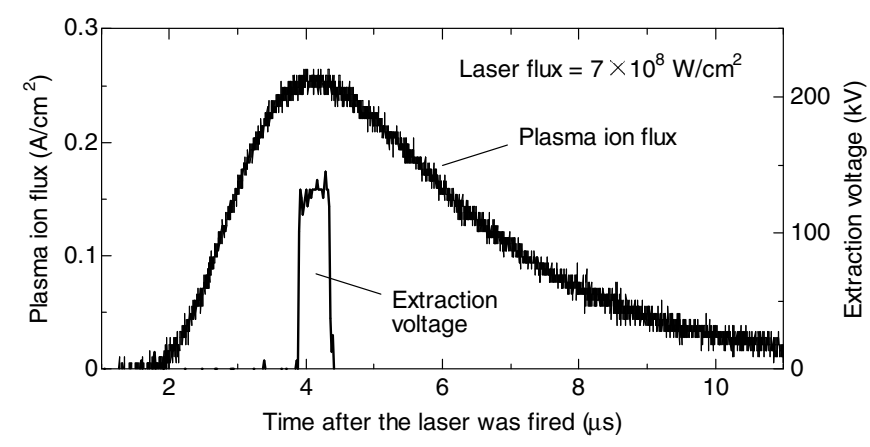

FIG. 3. Temporal variation of the plasma ion flux at the anode position. The laser was fired at time $=0$. The extraction voltage waveform is also plotted as a reference.

\section{RESULTS AND DISCUSSION}

\section{A. Plasma flux into the gap}

Figure 3 shows the temporal variation of the plasma ion flux at the anode position measured with the biased Faraday cup. For this measurement, the anode was removed. We see that at the anode position, $100 \mathrm{~mm}$ downstream from the $\mathrm{Cu}$ target, the duration of the plasma burst is roughly $\approx 10 \mu \mathrm{s}$. A peak of the ion flux was observed $\approx 4 \mu \mathrm{s}$ after the laser was fired. This time of flight corresponds to a kinetic energy of $130 \mathrm{eV}$ for $\mathrm{Cu}$ ions. As seen in the extraction voltage waveform included in the figure, the beam was extracted when the ion flux reached the maximum.

Figure 4 illustrates the peak ion flux measured at the anode position as a function of the laser flux onto the target. Depending on the laser flux, the averaged ion flux varied from 0 to $0.24 \mathrm{~A} / \mathrm{cm}^{2}$, although the kinetic energy of ions was almost constant even if the laser flux was changed. After attaching the anode sphere, the averaged ion current density $J_{\text {av }}$ without the extraction voltage was measured just behind the anode by the planar charge collector. The results are plotted in Fig. 4. $J_{\text {av }}$ was evaluated by the

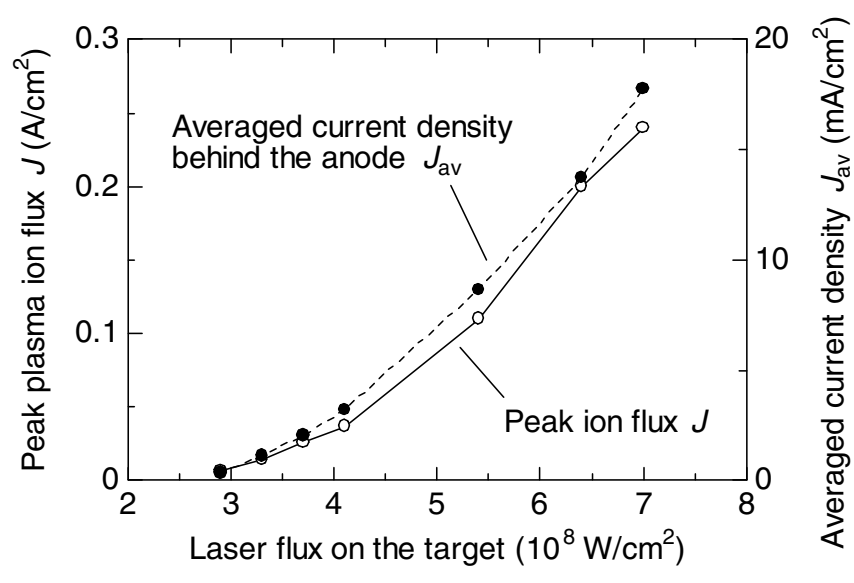

FIG. 4. Peak ion flux at the anode position as a function of the laser flux. 
measured total ion current divided by the effective ionemitting surface area $S_{\text {eff }}$ on the anode. We see that the maximum of $J_{\mathrm{av}}$ is $\approx 18 \mathrm{~mA} / \mathrm{cm}^{2}$.

The space-charge-limited ion current in a converging spherical geometry is given by [4]

$$
I_{\mathrm{SC}}=\frac{4 \varepsilon_{0}}{9} \sqrt{\frac{2 q e}{M}} \frac{4 \pi V^{3 / 2}}{\alpha^{2}},
$$

where $M$ and $q$ are the mass and the charge state of ions, respectively. The parameter $\alpha$ denotes the Langmuir function, which can be approximated by [4]

$$
\begin{aligned}
\alpha \approx & \gamma-0.3 \gamma^{2}+0.075 \gamma^{3}-0.0143182 \gamma^{4} \\
& +0.0021609 \gamma^{5}-0.00026791 \gamma^{6}+\cdots,
\end{aligned}
$$

where $\gamma \equiv \ln \left(R_{\mathrm{a}} / R_{\mathrm{c}}\right)$. The corresponding current density at the anode sphere is $I_{\mathrm{SC}} / 4 \pi R_{\mathrm{a}}^{2}=17 \mathrm{~mA} / \mathrm{cm}^{2}$ with $130 \mathrm{kV}$ applied across the diode. We see that the laser plasma can supply ion flux with intensities up to $I_{\mathrm{SC}}$ at the gap.

To investigate effects of the negative bias voltage on the anode mesh grid, the peak ion current into the gap was measured as a function of the bias voltage using the planar charge collector behind the anode. The cathode was removed for this measurement. The laser flux was fixed at $3.2 \times 10^{8} \mathrm{~W} / \mathrm{cm}^{2}$. The result is shown in Fig. 5 in conjunction with the data excluding the mesh grid on each hole in the anode. In this case, as for operations with the mesh grid, bias voltages $V_{\mathrm{b}} \approx-4 \mathrm{~V}$ is sufficient to completely suppress the flow of ions into the gap. On the other hand, we see that the bias voltage does not work if the mesh grid is not used. Even at $V_{\mathrm{b}}=0$, the ion current with the mesh is smaller than that without the mesh by a factor of $70 \%$. This can be attributed simply to the geometrical transmission of the grid $(\approx 40 \%)$.

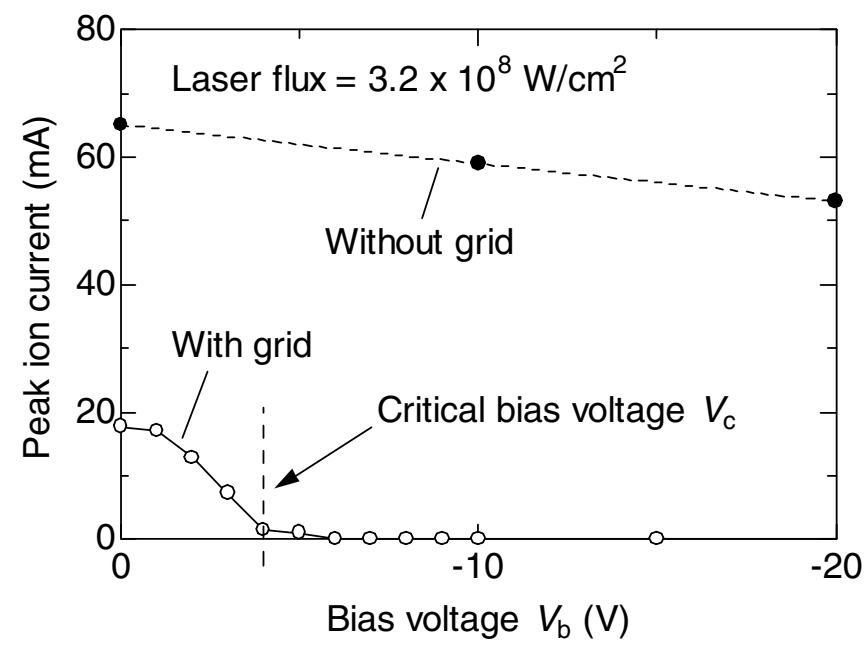

FIG. 5. Plasma ion current behind the anode as a function of the bias voltage with and without the anode mesh.

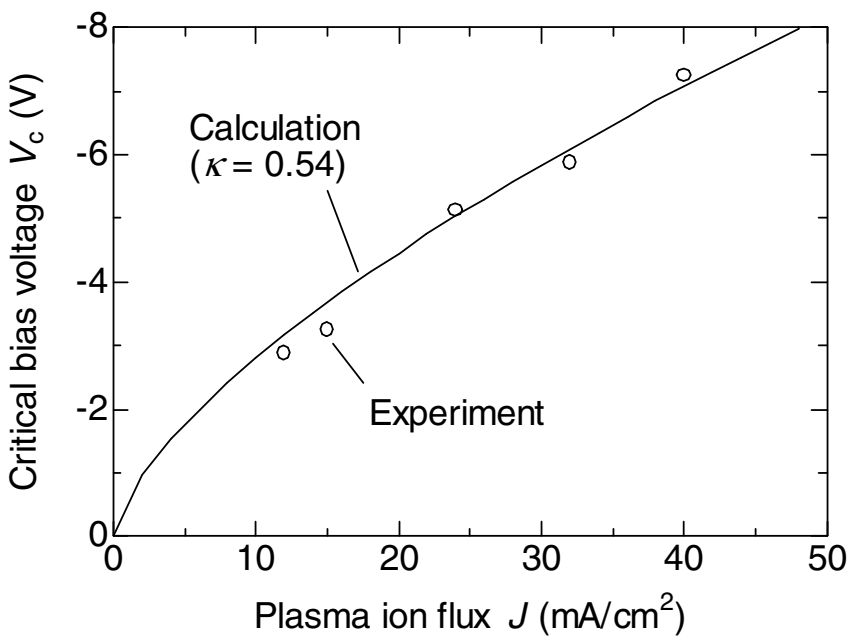

FIG. 6. Critical bias voltage $V_{\mathrm{c}}$ as a function of the plasma ion flux $J$. The curve represents a fit by Eq. (4).

Here we define the "critical bias voltage" $V_{c}$ as the voltage to reduce the ion current flow by $90 \%$. For instance, in the case of Fig. 5, we have $V_{\mathrm{c}}=-4$ V. Figure 6 illustrates the measured critical bias voltage $V_{\mathrm{c}}$ as a function of the peak ion flux supplied to the anode. In this measurement, the ion flux was adjusted by changing the laser intensity. As expected, the negative voltage required to suppress the plasma flow increases with the plasma flux.

The width of the ion sheath around the wires is determined by the balance between the plasma ion flux and space-charge-limited current flowing through the sheath [5,7]. These sheath regions work as a barrier for plasma electrons. If the sheath width reaches half of the mesh aperture, the plasma flow through the aperture can be completely stopped. Therefore the critical bias voltage $V_{\mathrm{c}}$ can be calculated by

$$
\sqrt{\frac{4 \varepsilon_{0}}{9}}\left(\frac{2 q e}{M}\right)^{1 / 4} \frac{V_{\mathrm{c}}^{3 / 4}}{\sqrt{J}}=\kappa\left(\frac{\delta}{2}\right),
$$

where $\delta$ denotes the aperture of the mesh grid $(=67 \mu \mathrm{m})$. The parameter $\kappa$ is the correction factor related to the geometrical configuration of the mesh. The solid line in Fig. 6 shows this result. The experimental result can be fit fairly well by the equation above if we take $\kappa=0.54$, which is smaller than unity. That is, the required bias voltage is lower than that obtained from the simple expectation that the plasma sheath width has to be larger than half of the mesh opening width.

\section{B. Extracted beam current waveform}

Figure 7 illustrates the effects of the bias voltage on the extracted beam current waveforms measured with the Faraday cup behind the extraction gap $(z=20 \mathrm{~mm}, z=$ 0 corresponds to the spherical center). The beam current density was evaluated by the measured beam current and 


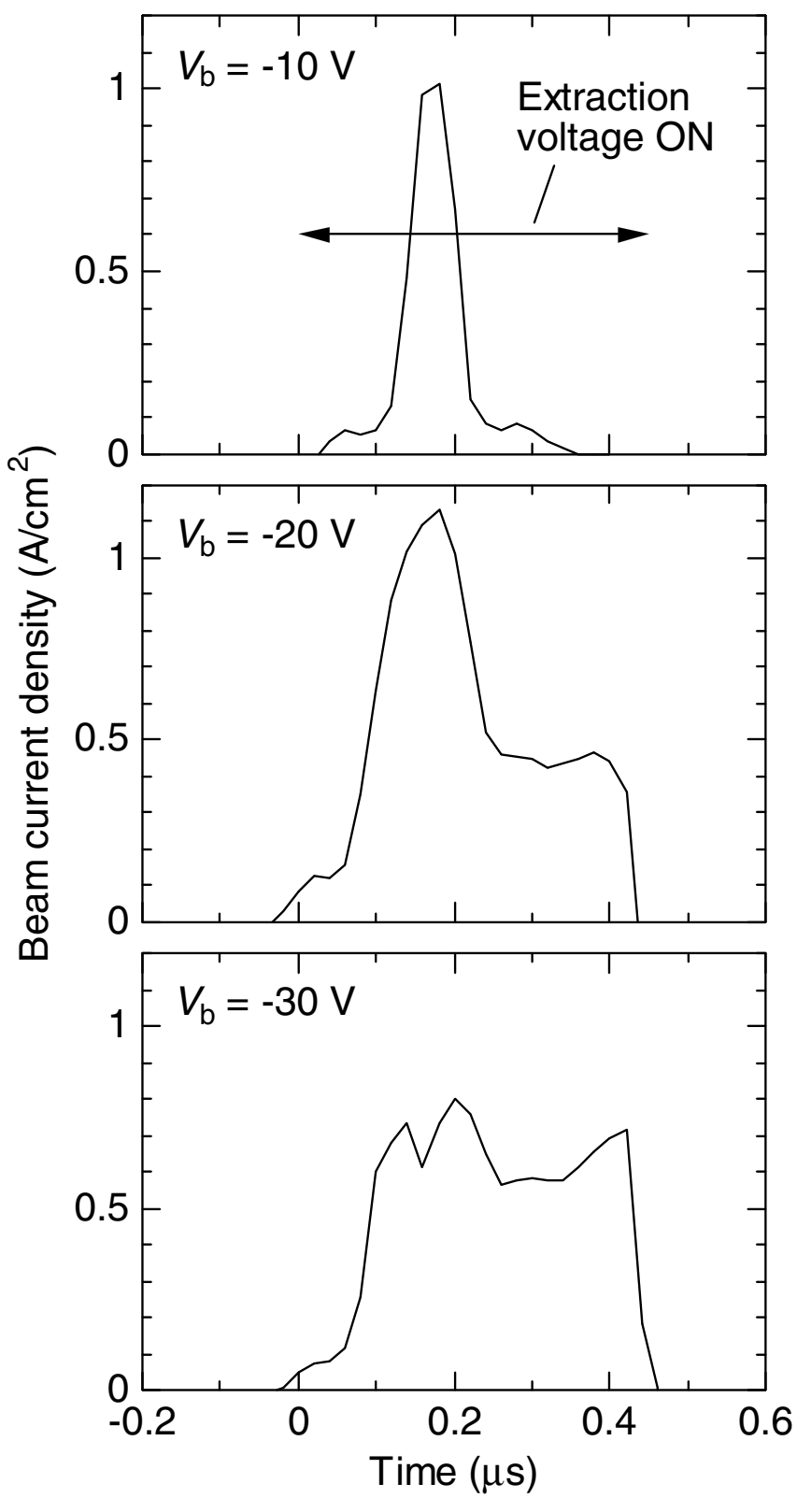

FIG. 7. Effects of the bias voltage $V_{\mathrm{b}}$ on the extracted beam current waveform measured at $z=20 \mathrm{~mm}$. The extraction voltage was applied at time $=0$.

the aperture size of the Faraday cup. The laser flux on the $\mathrm{Cu}$ target was fixed at $5.9 \times 10^{8} \mathrm{~W} / \mathrm{cm}^{2}$. The corresponding plasma ion flux at the anode position was $J \approx$ $0.2 \mathrm{~A} / \mathrm{cm}^{2}$. When the negative bias voltage is low $\left(V_{\mathrm{b}}=\right.$ $-10 \mathrm{~V})$, we see a spike of the beam current which can be attributed to the plasma prefill [6] in the gap. The instantaneous intensity of the spike is large since the effective extraction gap length at the plasma prefill mode is smaller than the real gap length. For a bias voltage of $-20 \mathrm{~V}$, we see a rectangular waveform appears after the spike component. This waveform shows that the confinement of the plasma is still not complete. However, when the negative bias voltage was raised to $-30 \mathrm{~V}$, the peak disappeared and an almost rectangular waveform was obtained. The rise time of this waveform was $\approx 100 \mathrm{~ns}$. Similar stabilizing effects of a biased grid on the beam waveforms have been observed for a laser ion source with a planar diode structure [8].

Using Eq. (4) and $J=0.2 \mathrm{~A} / \mathrm{cm}^{2}$, the critical bias voltage $V_{\mathrm{c}}$ can be estimated to be $-21 \mathrm{~V}$. This is consistent with the above experimental results, which indicates that $V_{\mathrm{c}}$ in this case lies between -20 and $-30 \mathrm{~V}$.

\section{Beam focusing by the spherical electrode structure}

Figure 8 shows the measured beam current density on the axis plotted as a function of the longitudinal position $z$ behind the gap, together with the numerical result. The laser flux was $7.0 \times 10^{8} \mathrm{~W} / \mathrm{cm}^{2}$. The bias voltage on the anode mesh grid was $-45 \mathrm{~V}$. In this case the ion current injected into the gap was $0.35 \mathrm{~A}$. The peak $\left(\approx 0.6 \mathrm{~A} / \mathrm{cm}^{2}\right)$ at $z \approx 10 \mathrm{~mm}$ suggests that the beam is focused around this position. These experimental results are consistent with the results of the numerical analysis.

Figure 9 shows calculated beam trajectories for different beam currents. The data of the beam transmission into the cathode sphere $T$ are inserted in the figure. The transmission $T$ is defined as the beam current behind the cathode divided by the current injected into the gap. Therefore $T$ is not simply the transmission through the cathode. The negative bias voltage is $-45 \mathrm{~V}$. If the beam current is 0 , an almost complete focusing is obtained at the center of the spherical structure. Nevertheless the beam transmission is only $61 \%$. This is mainly due to the initial drift velocity of the ions in the $z$ direction, which is not directed to the spherical center. If the beam current is increased, defocusing effect due to the strong space charge in the gap becomes substantial and the beam transmission decreases.

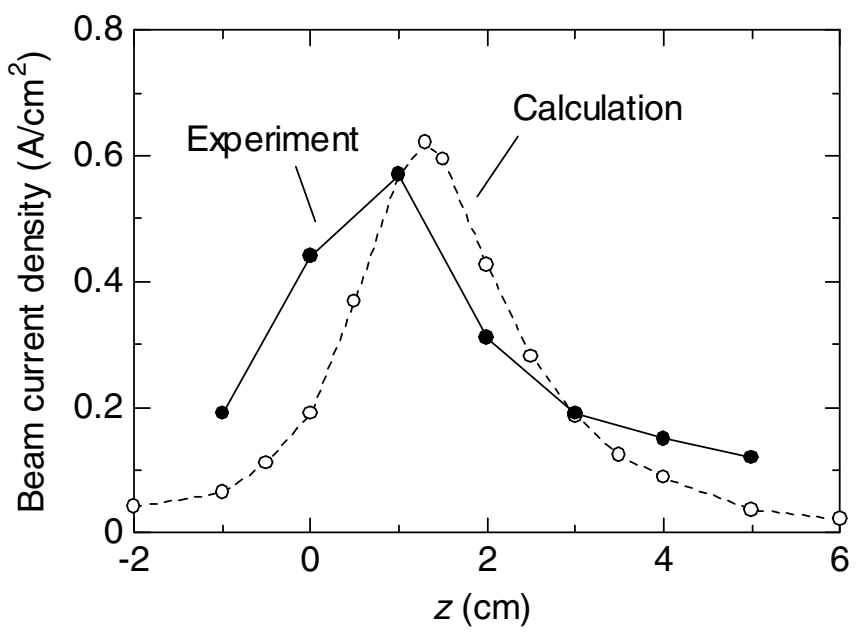

FIG. 8. Measured and calculated beam current density distributions along the beam axis. The position $z=0 \mathrm{~mm}$ corresponds to the spherical center of the diode structure. 

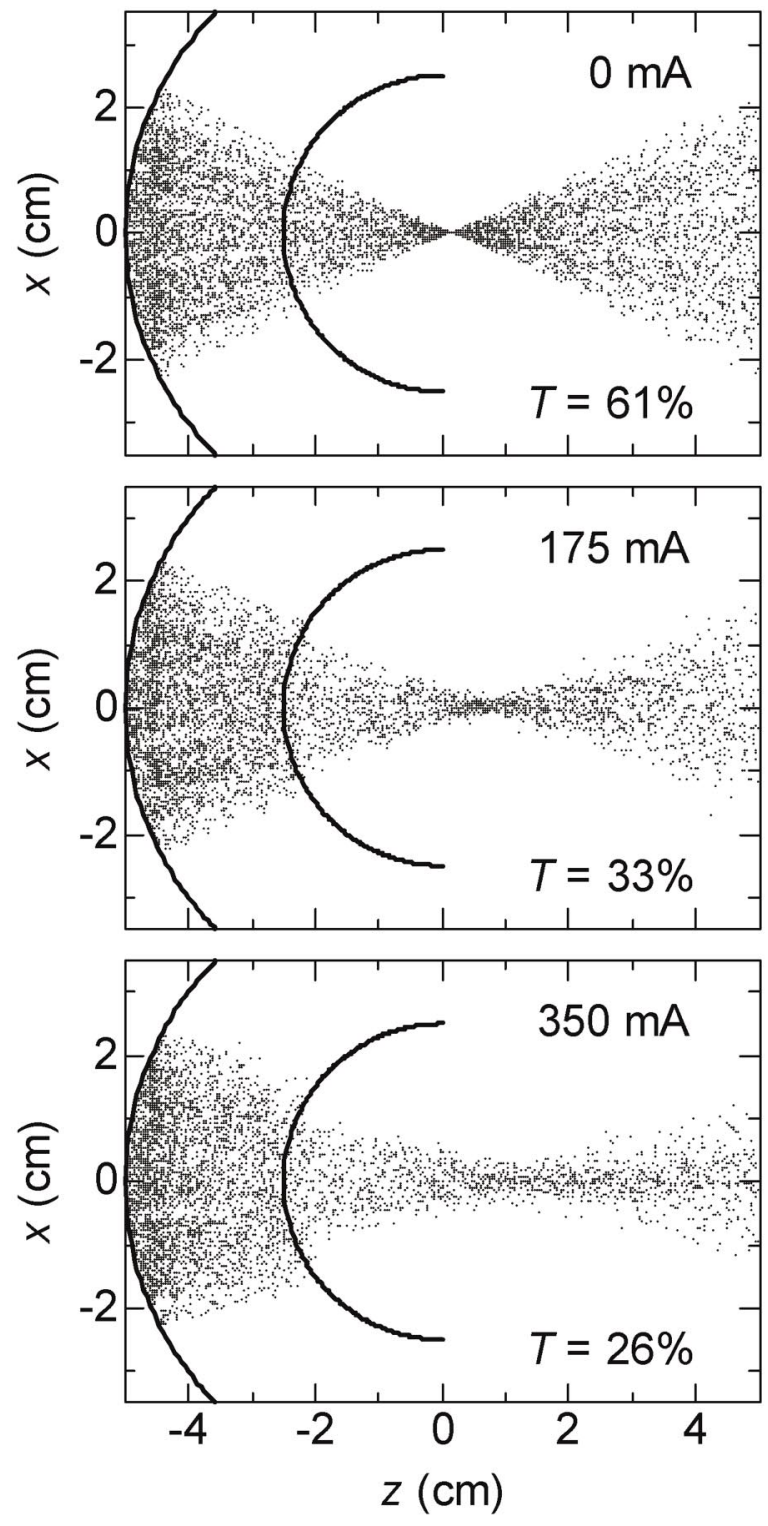

FIG. 9. Calculated beam trajectories for different initial beam currents. Also cross sectional view of the anode and cathode hemisphere are illustrated.
Also the focal position of the beam moves further downstream. For injected beam current of $0.35 \mathrm{~A}$, which corresponds to the experimental condition in Fig. 8, the focal position is $z \approx 10 \mathrm{~mm}$. In this case the beam current behind the cathode is only $91 \mathrm{~mA}$. During the calculation, we numerically observed some ions were pushed back to the source plasma by the strong longitudinal space-charge force, although this effect is not clearly seen in the figure. This is consistent with the fact that the ion flux from the plasma $\left(18 \mathrm{~mA} / \mathrm{cm}^{2}\right)$ is comparable to the space-chargelimited current density $\left(17 \mathrm{~mA} / \mathrm{cm}^{2}\right)$. If the whole cathode hemisphere was made of a metallic mesh, the transmission would be much better.

Figure 10 illustrates burn patterns on the burn paper put at different longitudinal positions. The conditions for the laser flux and the bias voltage are the same as above. We needed $\approx 100$ laser shots to clearly observe these patterns. One sees that the beam waist is at the center of the sphere $(z=0 \mathrm{~mm})$, whereas we have found a waist at $z \approx 10 \mathrm{~mm}$ by the measurement using the Faraday cup (Fig. 8). This discrepancy could be explained by inhomogeneity of the particle distribution in the beam. The waist radius is $\approx$ $5 \mathrm{~mm}$. The hexagonal pattern due to the configuration of the extraction holes is slightly visible only up to $z=$ $0 \mathrm{~mm}$. However, these patterns as well as the multihole structure have been smeared out for other longitudinal positions. Also in Fig. 10, we illustrate time-integrated beam profiles obtained by the numerical simulation. The beam exhibits a hexagonal structure before reaching the waist. This fine structure, however, is smeared out after passing through the waist. With the inclusion of this result, the behavior of the beam observed by the experiment is fairly well reproduced by the numerical analysis.

Figure 11 illustrates the beam emittance ellipse measured by the pepper-pot method at $z=20 \mathrm{~mm}$. The corresponding result of the numerical simulation is also plotted in the figure. The length of the vertical bars represents the full width of the beam emission angle at each transverse (horizontal, in this case) position. The normalized edge emittance was evaluated to be $\approx 3 \pi \mathrm{mm}$ mrad. The size

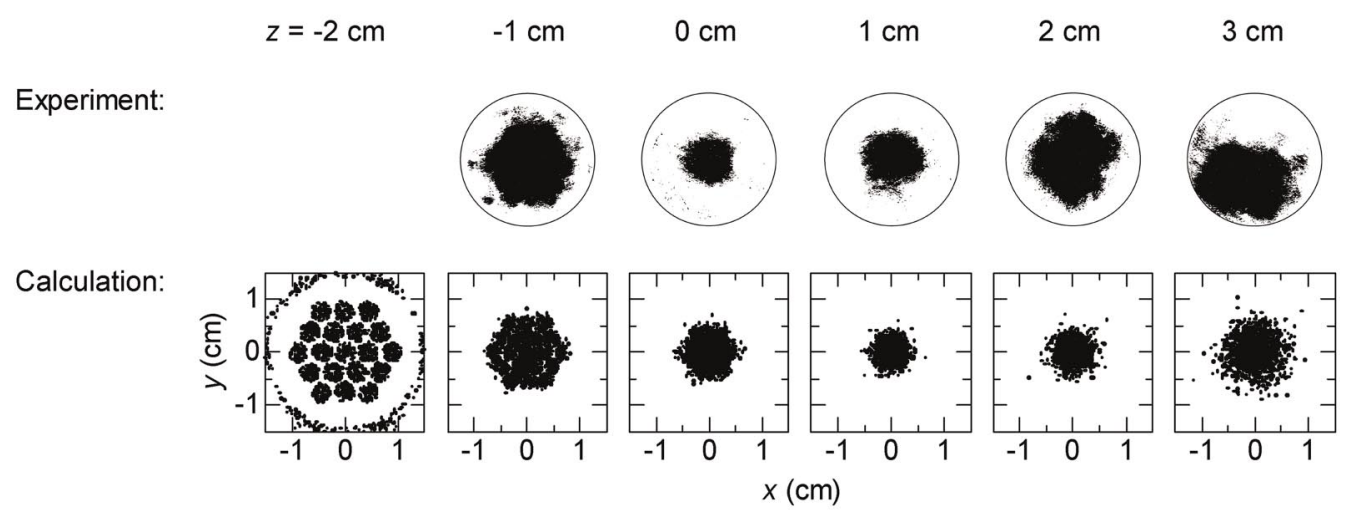

FIG. 10. Time-averaged beam profiles at different longitudinal positions obtained by the burn paper and the numerical method. 

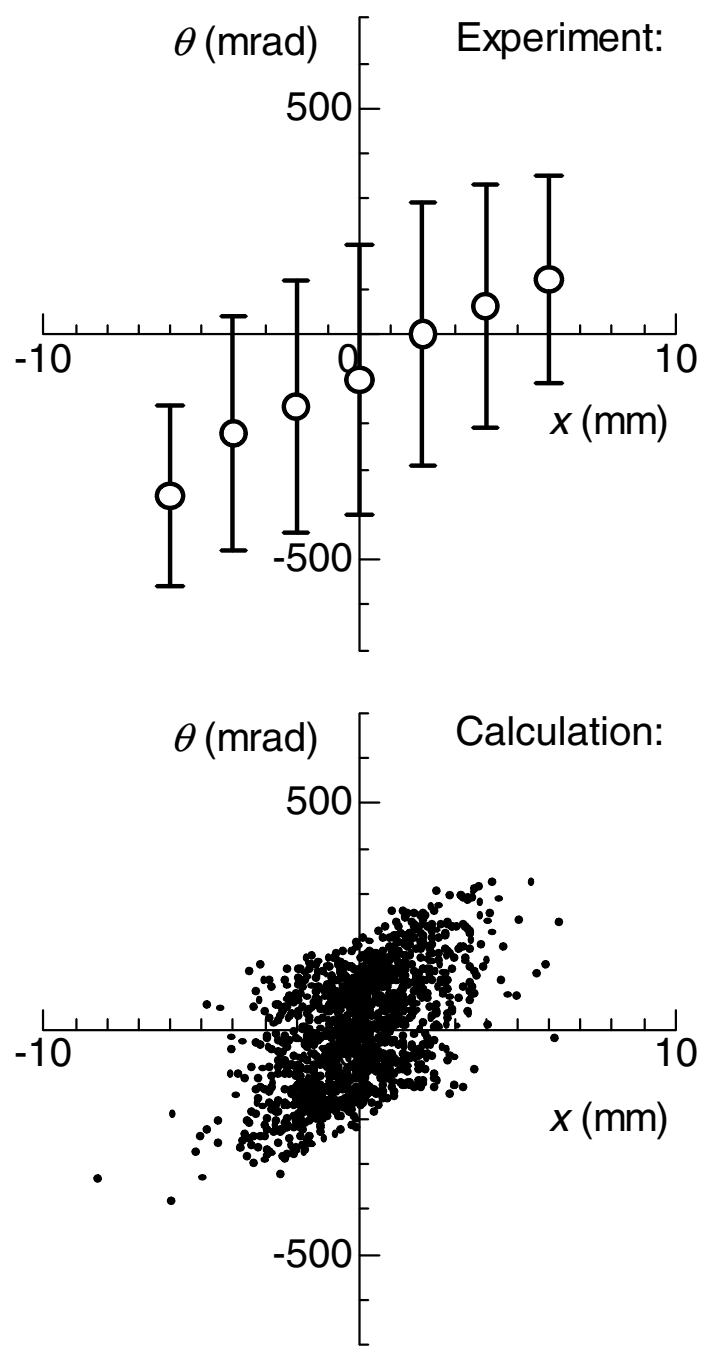

FIG. 11. Time-averaged horizontal emittance plots obtained by pepper-pot method (upper) and numerical calculation (lower).

of the ellipse is slightly larger than that obtained by the numerical simulation. This discrepancy can be explained by the shot-to-shot fluctuation of the beam condition. Nevertheless, the shape of the measured emittance ellipse is roughly consistent with the numerical result, although the emittance of each beamlet is assumed to be zero in the numerical calculation. Note that the pepper-pot pattern printed on the paper was complicated owing to the emission from multiple holes on the electrodes. Such microstructures were ignored in this evaluation. Hence the evaluated emittance includes large phase-plane areas without projectiles, like in the case where a large number of beamlets are merged to form a high-current single beam [15]. For the present work, we would not be able to detect any emittance degradation due to the scattering by the control grid, even if it existed. The corresponding beam brightness is $\approx 9 \mathrm{~mA} /(\mathrm{mm} \mathrm{mrad})$. This value is of the same order as a previous result obtained for a laser ion source with a single extraction aperture [6].

\section{CONCLUSIONS}

By means of the grid-controlled extraction, beam pulses with an almost rectangular waveform were extracted from a laser plasma. Minimally, the explosive divergence of the extracted beam due to the strong space-charge force could be avoided by the focusing action of the spherical diode structure. These experimental results have been fairly well reproduced by numerical simulations using a 3D particle code. With the help of this computer code, and by adjusting the spherical diode geometry we can find an appropriate beam-optics design matched to the next accelerator section. However, owing to divergence by the strong spacecharge force, as well as to the poor transmission through the cathode sphere, the beam current behind the cathode was limited to $\approx 0.1 \mathrm{~A}$. The output current can be improved by a few factors if the cathode sphere is replaced by a new one with higher geometrical transmission.

The degradation of the beam emittance due to fine mesh grids was not found to be serious for the proposed diode structure. It should be noted that the marginal beam emittance, which practically is the most important in the beam handling, is determined mainly by the spherical configuration of the beam-emitting surface and space-charge effects, and not by the initial emittance of each beamlet. As pointed out by Grote [15], this fact is advantageous for the optics design of the ion source system. Nevertheless to meet the requirement for ion sources for HIF drivers, the beam brightness should be further increased by several factors.

To obtain rectangular beam pulses with longer durations, the source plasma has to be further expanded before extraction. In this case, a more powerful laser is required since the ion flux into the gap is reduced by the additional expansion. Another induction cavity with a pulse duration of $\approx 10 \mu \mathrm{s}$ is also required.

\section{ACKNOWLEDGMENTS}

The authors thank M. Horiuchi for assistance in preparation of the experimental apparatus. H. Fukuda is also gratefully acknowledged for help in the experiment. This work was supported in part by a Grant-in-Aid for Scientific Research from the Japan Society for Promotion of Science, Sports and Culture (No. 11480122).

[1] J. W. Kwan, L. Ahle, D. N. Beck, F. M. Bieniosek, A. Faltens, D. P. Grote, E. Halaxa, E. Henestroza, W. B. Herrmannsfeldt, V. Karpenko, and T. C. Sangster, Nucl. Instrum. Methods Phys. Res., Sect. A 464, 379 (2001).

[2] J. Hovingh, V. O. Brady, A. Faltens, D. Keefe, and E. P. Lee, Fusion Technol. 13, 255 (1988).

[3] L. T. Perkins, J. W. Kwan, K. N. Leung, M. Rickard, and M. D. Williams, Rev. Sci. Instrum. 69, 1060 (1998). 
[4] S. Humphries, Jr., Charged Particle Beams (John Wiley \& Sons, New York, 1990).

[5] S. Humphries, Jr., C. Burkhart, and L. K. Len, in The Physics and Technology of Ion Sources, edited by I. G. Brown (John Wiley \& Sons, New York, 1989).

[6] M. Yoshida, J. Hasegawa, S. Fukata, Y. Oguri, M. Ogawa, M. Nakajima, K. Horioka, and M. Shiho, Rev. Sci. Instrum. 71, 1216 (2000).

[7] S. Humphries, Jr., C. Burkhart, S. Coffey, G. Cooper, L. K. Len, M. Savage, D. M. Woodall, H. Rutkowski, H. Oona, and R. Shurter, J. Appl. Phys. 59, 1790 (1986).

[8] M. Yoshida, J. Hasegawa, J. W. Kwan, Y. Oguri, M. Nakajima, K. Horioka, and M. Ogawa, Jpn. J. Appl. Phys. 42, 5367 (2003).

[9] T. Henkelmann, G. Korschinek, G. Belayev, V. Dubenkov, A. Golubev, S. Latyshev, B. Sharkov, A. Shumshurov, and B. Wolf, Rev. Sci. Instrum. 63, 2828 (1992).

[10] J. Collier, G. Hall, H. Haseroth, H. Kugler, A. Kuttenberger, K. Langbein, R. Scrivens, T. R. Sherwood,
J. Tambini, O. B. Shamaev, B. Yu. Sharkov, A. Shumshurov, S.M. Kozochkin, K. N. Makarov, and Yu. A. Satov, Rev. Sci. Instrum. 67, 1337 (1996).

[11] B. Sharkov, A. Shumshurov, I. Roudskoy, A. Kilpio, E. Shashkov, N. Kiselev, and P. Pashinin, Laser Part. Beams 17, 741 (1999).

[12] J. Hasegawa, M. Yoshida, Y. Oguri, M. Ogawa, M. Nakajima, and K. Horioka, Nucl. Instrum. Methods Phys. Res., Sect. B 161, 1104 (2000).

[13] J. Hasegawa, M. Yoshida, S. Fukata, Y. Oguri, and M. Ogawa, in Inertial Fusion Sciences and Applications 2001, edited by K. A. Tanaka, D. D. Meyerhoher, and J. Meyer-ter-Vehn (Elsevier, New York, 2002) p. 639.

[14] M. Yoshida, J. Hasegawa, S. Fukata, Y. Oguri, M. Ogawa, M. Nakajima, K. Horioka, S. Maebara, and M. Shiho, Nucl. Instrum. Methods Phys. Res., Sect. A 464, 582 (2001).

[15] D. P. Grote, E. Henestroza, and J. W. Kwan, Phys. Rev. ST Accel. Beams 6, 014202 (2003). 